Beyond Assessment of Quality of Life in Schizophrenia 

A. George Awad • Lakshmi N.P. Voruganti Editors

\section{Beyond Assessment of Quality of Life in Schizophrenia}




\section{Editors}
A. George Awad
Department of Psychiatry
University of Toronto and Humber
River Hospital
Toronto, Ontario
Canada

\author{
Lakshmi N.P. Voruganti \\ Department of Psychiatry \\ Oakville-Trafalgar Memorial Hospital \\ Oakville, Ontario \\ Canada
}

ISBN 978-3-319-30059-7 ISBN 978-3-319-30061-0 (eBook)

DOI 10.1007/978-3-319-30061-0

Library of Congress Control Number: 2016941540

(C) Springer International Publishing Switzerland 2016

This work is subject to copyright. All rights are reserved by the Publisher, whether the whole or part of the material is concerned, specifically the rights of translation, reprinting, reuse of illustrations, recitation, broadcasting, reproduction on microfilms or in any other physical way, and transmission or information storage and retrieval, electronic adaptation, computer software, or by similar or dissimilar methodology now known or hereafter developed.

The use of general descriptive names, registered names, trademarks, service marks, etc. in this publication does not imply, even in the absence of a specific statement, that such names are exempt from the relevant protective laws and regulations and therefore free for general use.

The publisher, the authors and the editors are safe to assume that the advice and information in this book are believed to be true and accurate at the date of publication. Neither the publisher nor the authors or the editors give a warranty, express or implied, with respect to the material contained herein or for any errors or omissions that may have been made.

Printed on acid-free paper

This Adis imprint is published by Springer Nature

The registered company is Springer International Publishing AG Switzerland 


\section{Foreword}

A serious, systematic interest in assessing quality of life experiences of persons with schizophrenia appeared in the professional literature nearly four decades ago. As in other areas of health outcome assessment, there was a stirring sense at that time that simply measuring parameters of illness did not adequately capture the impacts of illness and treatment on the lives of patients. More broadly, this illnessfocused approach did not adequately reflect the potential value of promoting and improving health. How do illness and treatment affect our ability to engage in all that life offers and our sense of well-being? These were heady questions, embedded in a broader sense of social activism, at a time when persons with schizophrenia lacked a voice about their care.

The context of this dawn of quality of life assessment provides context for this book. When quality of life assessment appeared on the scene, outcomes research in schizophrenia, as in most other disorders, stressed symptom reduction, symptom recurrence, and hospitalization. Strauss and Carpenter had only a few years earlier published their seminal work on the multiple dimensions of outcome in schizophrenia, showing that the course of symptoms, social functioning, and work impairment follow relatively independent trajectories. At the same time, leading policy voices raised critical social policy questions about how our changing systems of care were affecting persons with schizophrenia. Was deinstitutionalization a good thing? Were people with schizophrenia better off in the community? Quality of life assessment seemed a way to incorporate these health-related and humanistic concerns into the routine evaluation of treatments and social policies.

This early work on quality of life assessment in schizophrenia witnessed a proliferation of scales and primarily descriptive studies of quality of life experiences. At that time the focus was on a fairly broad notion of quality of life, capturing life experiences in many domains beyond health, such as housing, income, safety, interpersonal relationships, and neighborhoods. The advent of the newer generation of antipsychotic medications in the 1980s raised the hope these newer therapeutic agents with new modes of action and fewer (at least) different side effects would afford individuals with a better quality of life. We witnessed an intensified focus on quality of life outcomes in clinical trials. However, this hope did not pan out by and 
large. How could it be that these newer agents did not improve patient functioning and sense of well-being? This disappointment led to a much more critical consideration of the reliability and validity of self-reported quality of life, including how cognitive impairment inherent to schizophrenia may affect perception and judgment as well as how humans adapt psychologically to even the worst of circumstances to "make the best of things." Still, it appeared that at least from the perspective of patients, these new treatments were disappointing. Also, while quality of life assessment could document the adversity under which many persons with schizophrenia live, it was becoming clear that many of the vicissitudes of life - poverty, unemployment, substandard housing, violence, and social isolation - cannot be addressed directly by health care. Researchers realized that assessing the value of health care must be more focused. Hence the notion of "health-related quality of life" arose.

Despite all of these bumps in the road, assessment of quality of life has remained an important notion in health-care assessment. Why is this? This volume documents the many aspects of the history and current state of affairs of quality of life assessment. We see that it continues to evolve, both in how it is conceptualized and how it is measured. It seems embedded in our current notions of "patient-centered care" and "value-based treatment." It also certainly remains relevant today to considering the social circumstances of persons with schizophrenia living in our communities. One hopes that as our scientific understanding of the disorders that we now call schizophrenia grows and as new treatments emerge, we will truly be able to effectively incorporate quality of life into "value-based" assessments of "patientcentered" care. Finally we must retain the social values that led to interest in the quality of life of persons living with schizophrenia in the first place.

Baltimore, MD, USA

Anthony F. Lehman

October 2015 


\section{Preface}

Quality of life has emerged over the past few decades as an attractive and important concept that reflects a new image of health, viewed from a biopsychosocial perspective. The concept has been applied as an important attribute in patient care, in clinical outcome studies, and in health economic analysis.

In psychiatry and the mental health field, quality of life, particularly in schizophrenia, evolved as the result of increased concerns about the plight of the chronically mentally ill who were discharged into the community as a result of deinstitutionalization in the 1960s. The pioneering efforts of Professor Antony F. Lehman, who has kindly and graciously contributed a foreword to this book, is credited for the significant growth of research interest in quality of life in schizophrenia. Such heightened interest has led to the extensive development of measuring tools and clearly documented the poor state of quality of life among persons suffering from schizophrenia. As publications multiplied, the majority of them have concerned themselves with measurement and documentation of the state of quality of life, and only a few publications have gone beyond assessments into how quality of life can impact clinical care, become an important component in pharmaco and health economics, as well as impacting resource utilization and health policy decision-making. Such gaps, in spite of the initial enthusiasm, have gradually undermined the usefulness of such construct and led to noticeable erosion in clinical and research interest. As we believe that the construct of quality of life in schizophrenia continues to be important and relevant, the idea of this book became clearer. We believe that the construct of quality of life in schizophrenia needs to be revisited for the purpose of refining it conceptually, bridging the gaps and going beyond measurement to its significant applications in impacting clinical care and health economics. We have been fortunate to be able to sign in contributions from a number of leading experts in the field who share with us in the importance of quality of life assessment in schizophrenia going beyond assessments, and for that we are most grateful.

The topics covered in this book are constructed under four major parts. The first part deals with basic and conceptual issues, which includes a synopsis of psychopathology of schizophrenia issues, issues that slowed progress in the development of 
quality of life in schizophrenia, social cognition and its relevance to quality of life, cultural adaptation, and the role of culture in assessments of health-related quality of life in schizophrenia.

The second part includes a critical review of quality of life measures currently in use, modern approaches in scaling and the development of measuring tools, the electronic technology and its role in future advances in assessments of outcomes, quality of life assessments in the development and clinical trials of antipsychotics from the pharmaceutical industry perspective, and assessment of burden of care and quality of life of caregivers in schizophrenia.

The third part deals with important issues beyond assessment and includes a model for integration of quality of life assessments in care plans, quality of life as an outcome and mediator of other outcomes, use of quality of life measurement in cost-effectiveness and how to impact public health policy and resource allocation. A case study from India as a developing country illustrates the interplay of clinical, cultural, economic, and resources issues.

The final part provides an outline of future challenges in an effort to reinvigorate the construct of quality of life in schizophrenia.

Developing a book with multiple contributors, as in any extensive project, requires a high level of coordination and editorial management, which, in our case, has been efficiently provided by Ms. Pamela Walsh, for whom we acknowledge her assistance. We hope that this book can prove of value to clinicians, researchers, health- and pharmaco-economists, as well as health policy decision-makers. We hope this book is perceived in terms, not as conclusions, but as the beginning of an important conversation that needs to take place among all stakeholders, in an effort to invigorate such an important and relevant construct, not only for the benefit of the individual, but for the society as a whole.

Toronto, Canada

A. George Awad

Oakville, Canada

Lakshmi N.P. Voruganti 


\section{Contents}

\section{Part I Basic and Conceptual Issues}

1 Schizophrenia and Its Sequelae $\ldots \ldots \ldots \ldots \ldots \ldots \ldots \ldots \ldots$ Mary V. Seeman

2 Issues That Slowed Progress in Assessments of Health-Related Quality of Life in Schizophrenia

A. George Awad and Lakshmi N.P. Voruganti

3 Quality of Life, Cognition, and Social Cognition in Schizophrenia . . 25 Sofia Brissos, Guilherme Pereira, and Vicent Balanzá-Martinez

4 Conceptual Issues in Cultural Adaptation and the Role of Culture in Assessment of Health-Related Quality of Life in Schizophrenia . . . 53 Monika Vance, Elizabeth Pappadopulos, Richard Keefe, and Amir Kalali

\section{Part II Methodological Issues}

5 A Review of Quality-of-Life Assessment Measures in Schizophrenia:

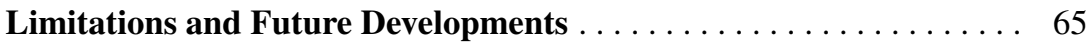
María Teresa Bobes-Bascarán, María Paz García-Portilla, Pilar A. Sáiz Martínez, Leticia García-Alvarez, Isabel Menéndez-Miranda, Susana Al-Halabí, María Teresa Bascarán, and Julio Bobes

6 Assessment of the Burden of Care and Quality of Life of Caregivers in Schizophrenia ............................. 79 Laurent Boyer, Karine Baumstarck, and Pascal Auquier

7 Electronic Technology and Advances in Assessment of Outcomes ... 95 Iris de Wit, Lieuwe de Haan, and Inez Myin-Germeys

8 Modern Psychometric Approaches to Analysis of Scales for HealthRelated Quality of Life . . . . . . . . . . . . . . . . . . . . 103 Jakob Bue Bjorner and Per Bech 
Part III Beyond Assessment of Quality of Life in Schizophrenia

9 Quality of Life as an Outcome and a Mediator of Other Outcomes in Patients with Schizophrenia

Anne Karow, Monika Bullinger, and Martin Lambert

10 Using Routine Quality of Life Assessment to Improve

Effectiveness of Community Mental Health Care . . . . . . . . . . . . . 145

Domenico Giacco and Stefan Priebe

11 Quality of Life Assessments in the Development and Clinical Trials of New Antipsychotics:

Pharmaceutical Industry Perspective .

Raimund Buller and Christophe Sapin

12 Quality of Life and Health Costs: The Feasibility of Cost-Utility Analysis in Schizophrenia

A. George Awad and Lakshmi N.P. Voruganti

13 Health-Related Quality of Life in Schizophrenia:

Health Policy and Resource Allocation. .

Frank Holloway and Jerome Carson

14 Beyond Assessment of Quality of Life in Schizophrenia:

Cultural, Clinical, and Research Perspectives from India,

a Case Study

Santosh K. Chaturvedi, M. Krishna Prasad, and Abhishek Pathak

Part IV Reinventing Quality of Life in Schizophrenia

15 Reinventing Quality of Life: Refining the Concept and Going Beyond Assessments. . . . . . . . . . . . . . . . . . . . . . . . . 219

A. George Awad 


\section{Contributors}

Susana Al-Halabí, PhD Centro de Investigación Biomédica en Red de Salud Mental, CIBERSAM, Oviedo, Spain

Pascal Auquier, MD, PhD Research Unit, Public Health, Chronic Diseases and Quality of Life, Aix - Marseille University, EA3279, Marseille, France

A. George Awad Department of Psychiatry, The Institute of Medical Science, University of Toronto, Toronto, ON, Canada

Vicent Balanzá-Martinez, MD, PhD Teaching Unit of Psychiatry, Department of Medicine, School of Medicine, La Fe University and Polytechnic Hospital, University of Valencia, CIBERSAM, Valencia, Spain

María Teresa Bascarán, MD, PhD Centro de Investigación Biomédica en Red de Salud Mental, CIBERSAM, Oviedo, Spain

Karine Baumstarck, MD, PhD Research Unit, Public Health, Chronic Diseases and Quality of Life, Aix - Marseille University, EA3279, Marseille, France

Per Bech, MD, PhD Psychiatric Research Unit, CCMH, Mental Health Centre North Zealand, Hillerød, Denmark

Jakob B. Bjorner Patient Insights, Lincoln, RI, USA

Department of Public Health, University of Copenhagen, Copenhagen, Denmark National Research Centre for the Working Environment, Copenhagen, Denmark

Julio Bobes, MD, PhD Department of Psychiatry, University of Oviedo, Oviedo, Spain

Centro de Investigación Biomédica en Red de Salud Mental, CIBERSAM, Oviedo, Spain

María Teresa Bobes-Bascaran, PhD Centro de Investigación Biomédica en Red de Salud Mental, CIBERSAM, Valencia, Spain 
Laurent Boyer, MD PhD Research Unit, Public Health, Chronic Diseases and Quality of Life, Aix-Marseille University, EA3279, Marseille, France

Sofia Brissos, MD Centro Hospitalar Psiquiátrico de Lisboa (Lisbon's Psychiatric Hospitalar Centre), Lisbon, Portugal

Raimund Buller, MD Clinical Development, Lundbeck, Issy-les-Moulineaux, France

Monika Bullinger, PhD Institute for Medical Psychology, Centre for Psychosocial Medicine, University Medical Centre Hamburg-Eppendorf, Hamburg, Germany

Jerome Carson School of Education and Psychology, University of Bolton, Bolton, UK

Santosh K. Chaturvedi, MD, FRCPsych Department of Mental Health Education, National Institute of Mental Health \& Neurosciences, Bangalore, India Department of Psychiatry, National Institute of Mental Health \& Neurosciences, Bangalore, India

Leticia García-Álvarez, PhD Department of Psychiatry, University of Oviedo, Oviedo, Spain

Centro de Investigación Biomédica en Red de Salud Mental, CIBERSAM, Oviedo, Spain

María Paz García-Portilla, MD, PhD Department of Psychiatry, University of Oviedo, Oviedo, Spain

Centro de Investigación Biomédica en Red de Salud Mental, CIBERSAM, Oviedo, Spain

Domenico Giacco, MD, PhD Unit for Social and Community Psychiatry, WHO Collaborating Centre for Mental Health Services Development, Queen Mary University of London, East London NHS Foundation Trust, Newham Centre for Mental Health, London, UK

Lieuwe de Haan Psychotic Disorders, AMC Academic Psychiatric Centre, Amsterdam, The Netherlands

Early Psychosis, AMC Psychiatry, Amsterdam, AZ, The Netherlands

Frank Holloway, FRCPsych South London and Maudsley NHS Foundation Trust, Beckenham, UK

Maudsley Hospital, Denmark Hill, London, UK

Amir Kalali, MD Neuroscience Center of Excellence, Quintiles, San Diego, CA, USA

Anne Karow, MD Department of Psychiatry and Psychotherapy \& Department of Child and Adolescent Psychiatry, Centre for Psychosocial Medicine, University Medical Centre Hamburg-Eppendorf, Hamburg, Germany 
Richard Keefe, PhD Psychiatry \& Behavioral Sciences and Psychology \& Neuroscience, Duke University Medical Center, Durham, NC, USA

Martin Lambert, MD Department of Psychiatry and Psychotherapy \& Department of Child and Adolescent Psychiatry, Centre for Psychosocial Medicine, University Medical Centre Hamburg-Eppendorf, Hamburg, Germany

Pilar A. Sáiz Martínez, MD, PhD Department of Psychiatry, University of Oviedo, Oviedo, Spain

Centro de Investigación Biomédica en Red de Salud Mental, CIBERSAM, Oviedo, Spain

Isabel Menendez-Miranda, MD PhD Mental Health Services of Asturias, Oviedo, Spain

Inez Myin-Germeys Department of Neuroscience, Centre for Contextual Psychiatry, KU Leuven, Belgium

Department of Psychiatry and Psychology, School of Mental Health and Neuroscience, Maastricht University, Maastricht, The Netherlands

Elizabeth Pappadopulos, PhD Pfizer Inc, New York, NY, USA

Abhishek Pathak, MD Department of Psychiatry, National Institute of Mental Health \& Neurosciences, Bangalore, India

Guilherme Pereira, MD Centro Hospitalar Psiquiátrico de Lisboa (Lisbon's Psychiatric Hospitalar Centre), Lisbon, Portugal

M. Krishna Prasad, MD Department of Psychiatry, National Institute of Mental Health \& Neurosciences, Bangalore, India

Stefan Priebe, DiplPsych, DrMedHabil, FRCPsych Unit for Social and Community Psychiatry, WHO Collaborating Centre for Mental Health Services Development, Queen Mary University of London, Newham Centre for Mental Health, London, UK

Christophe Sapin, MSc Global Analytics, Lundbeck A/S, Issy-les-Moulineaux, France

Mary V. Seeman Department of Psychiatry, The Institute of Medical Science, University of Toronto, Toronto, ON, Canada

Monika Vance Santium Mental Health, Vaughan, ON, Canada

Lakshmi N.P. Voruganti, MD, Msc, PhD Department of Psychiatry, Oakville-Trafalgar Memorial Hospital, Oakville, ON, Canada

Iris de Wit, MSc Early Psychosis, AMC Psychiatry, Amsterdam, AZ, The Netherlands

Department Early Psychosis, AMC, Academic Psychiatric Centre, Amsterdam, AZ, The Netherlands 